Research Article

\title{
Long Cycle Life TiC Anode Fabricated via High-Energy Ball Mill for Li-Ion Battery
}

\author{
Yuduo Ren 1 and Shiting Zhang \\ Jilin Jianzhu University, School of Materials Science and Engineering, Jilin 132001, China
}

Correspondence should be addressed to Yuduo Ren; renyd_work@sina.com

Received 30 July 2020; Revised 26 September 2020; Accepted 5 October 2020; Published 22 October 2020

Academic Editor: Renyun Zhang

Copyright () 2020 Yuduo Ren and Shiting Zhang. This is an open access article distributed under the Creative Commons Attribution License, which permits unrestricted use, distribution, and reproduction in any medium, provided the original work is properly cited.

\begin{abstract}
Nano-TiC and nano-WC anodes for Li-ion battery were manufactured by high-energy ball milling. Pure titanium powder and toluene are mixed with a high-energy ball mill to prepare $\mathrm{TiC}$ powder. The powder is calcined at $750^{\circ} \mathrm{C} / 1 \mathrm{~h}$ and secondary ball milled to make a negative electrode for lithium-ion battery. The phase composition and micromorphology of TiC powder are analyzed and observed, and the charge-discharge cycle performance of TiC anode material is tested. The results show that there are $\mathrm{TiH}_{2}$ and WC impurities in the product after primary ball milling. After calcination and secondary ball milling, TiH impurities are removed and the $\mathrm{TiC}$ grain size is refined, and $\mathrm{TiC}$ powder is obtained with a grain size of $12.5 \mathrm{~nm}$. The specific discharge capacity of the $\mathrm{TiC}$ anode is stable during the long cycle discharge. When the current density is $1 \mathrm{~A} / \mathrm{g}$, the specific discharge capacity can still be maintained at $110 \mathrm{mAh} / \mathrm{g}$ after 3000 cycles. The results show that TiC anode materials have excellent long-cycle performance and could be used as the frame material of Si anode materials. Nano-WC powders are prepared by a ball milling method to investigate the effect of WC impurities on the performance of TiC lithium batteries. The charge and discharge capacity at $0.5 \mathrm{~A} / \mathrm{g}$ current density is similar to that of TiC anode. After 2000 cycles, the discharge-specific capacity is about $100 \mathrm{~mA} / \mathrm{g}$, which is slightly lower than $\mathrm{TiC}$, and the final capacity is maintained at $230 \mathrm{~mA} / \mathrm{g}$, but its low discharge capacity affects the performance of the TiC battery after a long ball milling. The results show that the performance of the $\mathrm{TiC}$ anode after the first $50 \mathrm{~h}$ of ball milling is poor. The main reason is the agglomeration of TiC nanoparticles.
\end{abstract}

\section{Introduction}

Recently, there have been lots of attempts to seek alternative materials for the anode of Li-ion battery (LIB) to achieve better cycling performance including long-term cyclability and stable Li-ion storage $[1,2]$. General methods to implement this intent is to set up a nanostructure of core/shell [3] or to produce a thermal stable matrix [4-6]. Transition metal carbide (TMC) has a very stable structure and is widely used as structural materials [7]. Among them, TiC have attracted much attention because of its high stiffness, high electrical, and high thermal conductivity. Researchers use $\mathrm{TiC}$ as a matrix structure for silicon. Composite of $\mathrm{Si} / \mathrm{TiC}$ can form an active/inactive system [8]; this structure presents higher capacities and more efficient cyclability. Although Si has a high theoretical capacity, due to its severe volume expansion caused by Li-ion insertion/deinsertion, the storage of LIB will eventually drop within 400 cycles [5]. This leads to the longterm cyclability of $\mathrm{Si} / \mathrm{TiC}$ anode that is a shortcoming for LIB. Otherwise, dispersing the active particle homogeneously is difficult to achieve. The aggregated $\mathrm{Si}$ is fatal for the battery. Therefore, anode prepared only by $\mathrm{TiC}$ with a similar active/inactive system could be a suitable solution. However, the pure $\mathrm{TiC}$ electrode that served as anode for LIB is rarely reported.

On the other hand, the particle size of anode material is a dominant factor that affects the performance of LIB. The nanoscale particle could shorten the route of Li-ion during transportation and provide a larger surface area to allow more Li-ion to adhere to the anode $[4-6,9]$. There were various methods for manufacturing nanocomposite anode, such as mechanical milling [10], Self-propagation Hightemperature Synthesis (SHS) [11], and Chemical Vapor Deposition (CVD) [12]. Among these methods, high- 
energy ball milling is much attractive because it is a simpler and more rapid way to produce nanosize powder with less impurity compare to chemical methods.

In this study, we used ball-milled $\mathrm{TiC}$ powder after a series of homogeneous treatment as an anode to assemble secondary LIB. The phase composition and micromorphology of anode material changes before and after cycling were observed. The cyclic performance was tested with longterm cycling, combined with the morphology images. The cycling performance of $\mathrm{TiC}$ anode was discussed.

In the process of mechanical alloying, it is inevitable that the ball milling medium will be peeled off. After the ball milling medium was stripped, it would participate in the ball milling together with the raw materials during the ball milling process, which will have a certain impact on the various properties of the ball milling products. Therefore, the nanoWC anode was prepared in the same way as the nano-TiC powder, then its electrochemical performance was tested, and the effect of WC impurities on the performance of the TiC anode was analyzed.

\section{Materials and Methods}

The raw materials used in this study were $99.9 \%$ pure Ti powder and analytically pure toluene. Powder and liquid were mixed into a fine slurry with a mass ratio of $3: 1$ and then milled by a planetary ball mill (FRITSCH P4 Germany) for selected time with Ar gas. The weight ratio of ball to materials was $20: 1$. After the primary mill process, the product was vacuum annealed at $750^{\circ} \mathrm{C}$ for $1 \mathrm{~h}$ and then reball milled for $20 \mathrm{~h}$. At last, the powder was loaded in an argon atmosphere and prepared for LIB assemble. Phase structure and transition during ball milling were characterized by X-ray diffraction $\left(\mathrm{Cu}, \mathrm{K}_{\alpha}\right.$ radiation, Rigaku SmartLab). The re-BMed powders were investigated by a transmission electron microscope (TEM, JEM-2010).

For electrochemical tests, the $\mathrm{TiC}$ powder treated by 2 step BM painted on the $\mathrm{Cu}$ chip was used as anode, and a counter and reference electrode was used as the cathode. Two electrode coin type cells (CR2032) were assembled in an argon atmosphere. A $1 \mathrm{M} \mathrm{LiPF}_{6}$ in a mixture of ethylene carbonate and dimethyl carbonate was used as the electrolyte, and a polypropylene film was used as the separator. The galvanostatic charge-discharge tests were conducted on a CT2001 battery program control system at room temperature with the cut-off voltage of 0.01 and $3 \mathrm{~V}$ under a chosen current density. Cyclic voltammetry measurements were performed on a CHI660D Electrochemical Workstation with a scan rate of $0.1 \mathrm{mVs}^{-1}$ between 0 and $3.5 \mathrm{~V}$.

\section{Result and Discussion}

3.1. Characterization of the Nanocomposite. Figure 1(a) shows the XRD patterns of TiC powder after a series period of the ball milling process. The main composition of asmilled powder was the $\mathrm{TiC}$ of characteristic peaks (111) (200) (220) (311) (222). The board peak indicated the formation of small size grains. The grain size was about $4.9 \mathrm{~nm}$ calculated by the Scherrer equation. A little peak that appears at about $40^{\circ}$ was the $\mathrm{TiH}_{2}$ phase. $\mathrm{TiH}_{2}$ was synthesized by Ti and $\mathrm{H}$ from residues of toluene, which was harmful to the battery performance. In order to eliminate the effect of impurity and residue, the powder was annealed at $750^{\circ} \mathrm{C}$ for $1 \mathrm{~h}$. $\mathrm{TiH}_{2}$ was decomposed into $\mathrm{Ti}$ and $\mathrm{H}$; this transition was able to produce a fresh $\mathrm{Ti}$ surface. The XRD pattern of the powder after the anneal treatment represented clearly pure $\mathrm{TiC}$ without the $\mathrm{TiH}_{2}$ phase. Besides, grain size grew to $26.1 \mathrm{~nm}$ due to the crystallization during the anneal process; moreover, sample powder was re-BMed for $20 \mathrm{~h}$ to gain finer grains. The pattern of Figure 1(c) showed comparatively pure $\mathrm{TiC}$, and grain size was approximately $12.5 \mathrm{~nm}$. It was finer enough for an anode material. For ease of interpretation, we named the $\mathrm{TiC}$ as-milled $40 \mathrm{~h}$ with anneal and re-BMed as $\mathrm{TiC}-40$ in the following explanation. In addition, a small amount of WC can be found after the anneal process, which was an inevitable impurity peeling off from mill balls. Considering the balance between finer grain and less impurity, TiC40 was chosen as a start material. Figure 2 presents the bright-field TEM images of TiC-40 nanoparticle. The grain size of single $\mathrm{TiC}$ particles was 10-20 nm. Severe agglomeration can be noticed, which was very common in researches about mechanical alloy $[13,14]$. Nanoparticle with higher surface energy had a trend of agglomerating to a bigger particle. It was a disadvantage for the battery performance of an anode material.

3.2. Discharge and Charge Performance. The cycling performance of TiC-40 anode was investigated by galvanostatic charge/discharge cycling at 0.01-3 V. Figure 3(a) shows the charge and discharge curve of TiC-40 for 1st, 5th, 10th, 20th, and 30th cycles at $1 \mathrm{Ag}^{-1}$. The anode showed a high discharge capacity of $225.9 \mathrm{mAhg}^{-1}$ and a charge capacity of $95.9 \mathrm{mAhg}^{-1}$. The Coulombic efficiency was $42.5 \%$. The irreversible capacity was $130.1 \mathrm{mAhg}^{-1}$. Inferentially, this was caused by the formation of the SEI layer. With further cycling to 1400th as shown in Figure 2(b), the discharge capacity increased gradually to $138 \mathrm{mAhg}^{-1}$ and the Coulombic efficiency held at $99.5 \%$. Figure 3(c) shows the cycling performance of $\mathrm{TiC}-40$ at the current density of $1 \mathrm{Ag}^{-1}$. The discharge capacity of the 1 st cycle was $225.9 \mathrm{mAhg}^{-1}$ and then decreased rapidly to $102.5 \mathrm{mAhg}^{-1}$ in the 2 nd cycle. This was caused by the SEI layers that emerged on the surface of the anode material, when the SEI layer was stable, the discharge capacity reached the lowest point $\left(62.5 \mathrm{mAhg}^{-1}, 30\right.$ th cycle). As the cycling continues, the capacity reached to the high point (142.5 $\mathrm{mAhg}^{-1}, 2010$ th cycle) and then declined gradually. At 3000th, the capacity remained at $110 \mathrm{mAhg}^{-1}$, which demonstrated an excellent long-term cyclic performance of $\mathrm{TiC}$ anode.

To further investigate the long cycle life of $\mathrm{TiC}$ anode, Figure 3(d) shows the cycling performance of $\mathrm{TiC}-40$ anode at a certain current rate. After 3000 cycles at $1 \mathrm{Ag}^{-1}$, the current density resets to $0.05 \mathrm{Ag}^{-1}$; the cycling performance was tested at a current rate of $0.25 \mathrm{Ag}^{-1}, 0.5 \mathrm{Ag}^{-1}, 0.75 \mathrm{Ag}^{-1}, 1 \mathrm{Ag}^{-1}$, $1.5 \mathrm{Ag}^{-1}$, and $2 \mathrm{Ag}^{-1}$ for 20 cycles each. And the current density returned to $0.05 \mathrm{Ag}^{-1}$ in the last 20 cycles. The Coulombic efficiency during 3000 cycles was also tested. The discharge capacity at a series of current density was 277.1, 212, 173.7, 


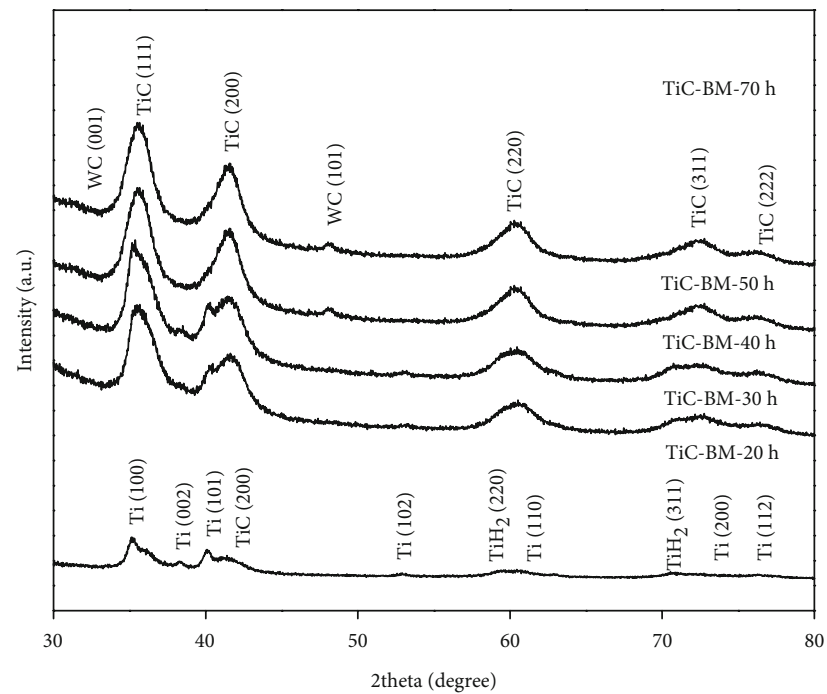

(a)

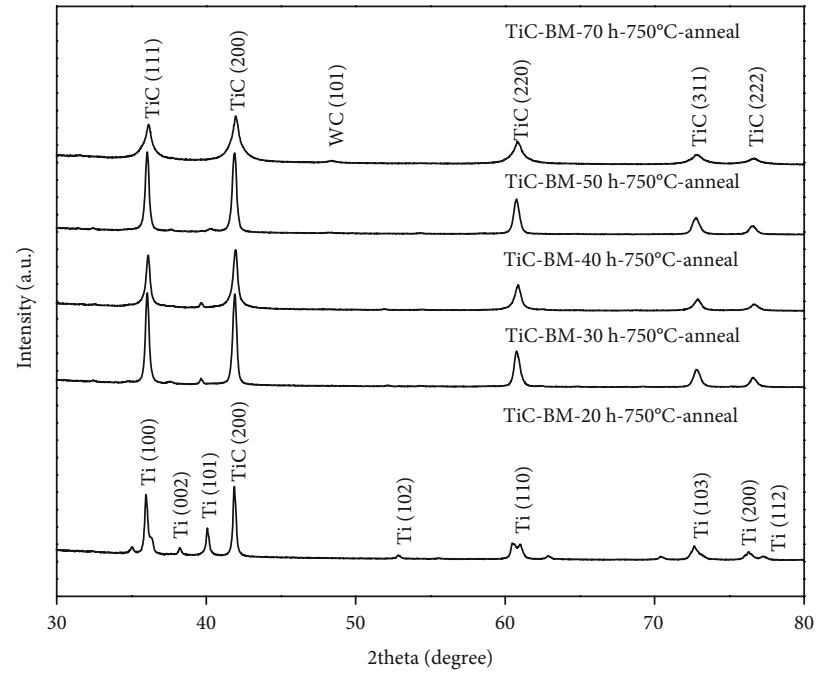

(b)

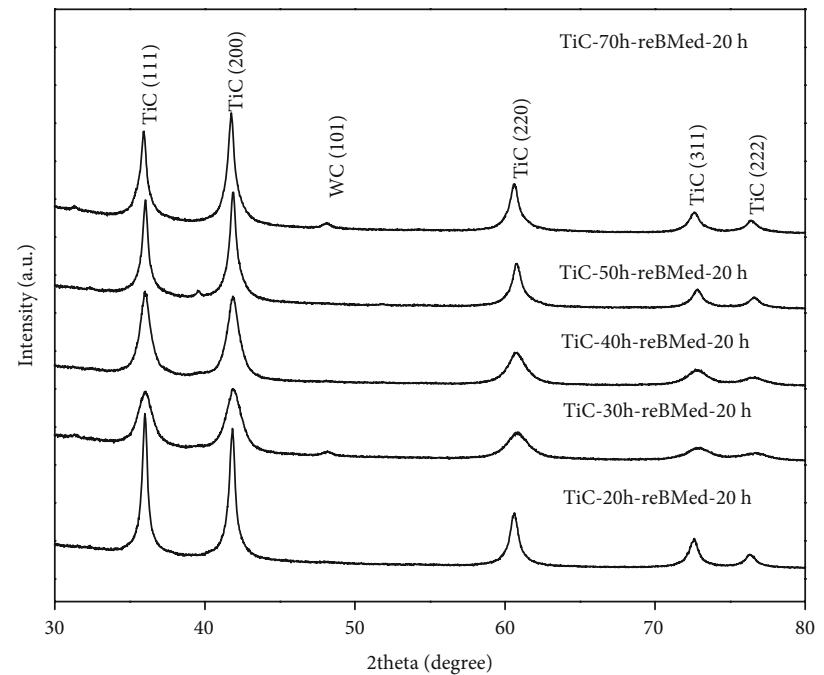

(c)

FIGURE 1: XRD pattern for nano-TiC obtained by (a) ball milling, (b) annealing after BM, and (c) re-BM. 


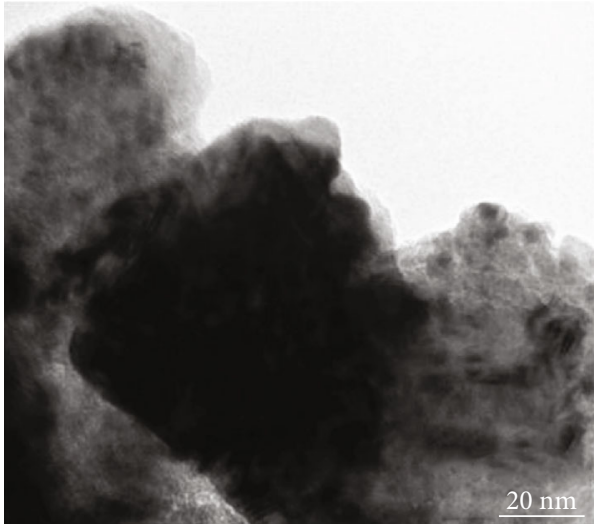

(a)

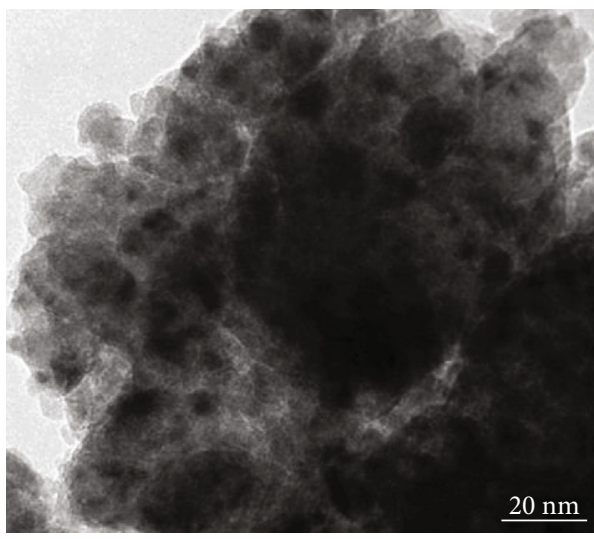

(c)

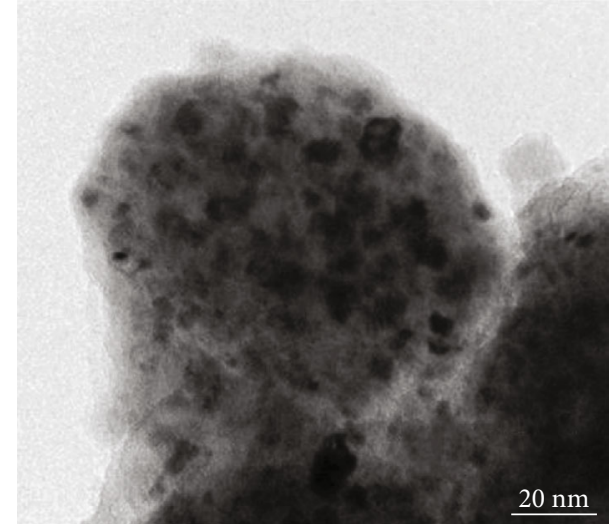

(b)

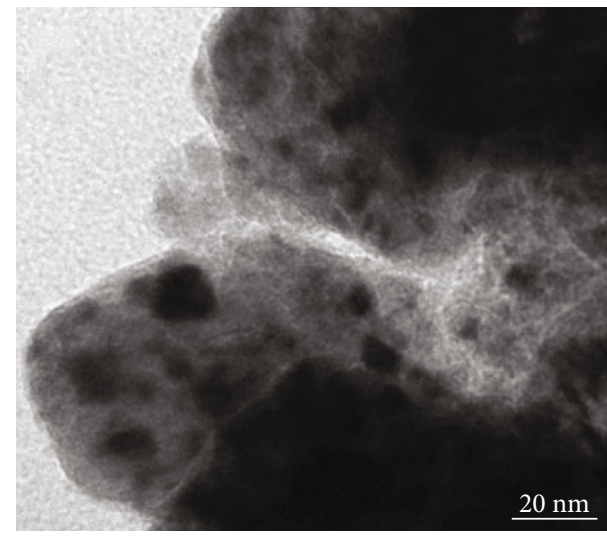

(d)

Figure 2

$152.1,134.4,113.2,96.1$, and $298.8 \mathrm{mAhg}^{-1}$, respectively. In the last 20 cycles, the capacity was even a little higher than the first 20 cycles, which showed excellent long-term cycling performance. The Coulombic efficiency was $96.9 \%$ at first, maintains a $98 \%$ average, and reaches to $99.3 \%$ at 160 cycles. These results indicated that $\mathrm{TiC}$ anode synthesized by BM has excellent high rate performance and long cycling stability.

To investigate the oxidation-reduction behavior of the discharge process, Figure 3(e) shows the cyclic voltammograms of TiC-40 for a series of cycles. Two oxidation peaks were found during the discharge process, and the intensity of the peaks enhanced as the cycling number increased. The oxidation peak that emerged at $3 \mathrm{~V}$ probably indicated the reaction between $\mathrm{Li}$ and graphite which was not involved in the synthesis of the $\mathrm{TiC}$ reaction. And the oxidation peak that appears at $0 \mathrm{~V}$ could be attributed to the formation of the SEI layer. The area of the closed curve increased as more anode materials are involved in the reaction, which is corresponding to the capacity-cycle data.

3.3. Effect of WC Impurities on the Performance of TiC Anode. In this part of the study, nano-WC powder was prepared, which was basically prepared in the same way as $\mathrm{TiC}$, and the raw materials were $99 \%$ pure $\mathrm{W}$ powder and toluene. $\mathrm{W}$ powder and toluene solution were mixed in a glove box and then loaded into a planetary ball mill. In order to exclude the influence of other impurities in the process of highenergy ball milling, WC grinding tanks and balls were used, in which the mass ratio of the ball to $\mathrm{W}$ powder and toluene was $10: 1$, the ball mill speed was set to $600 \mathrm{rpm}$. XRD pattern for nano-WC powder obtained by ball milling is shown in Figure 4.

During the ball milling process of $\mathrm{W}$ powder in toluene, the phase change process was similar to that of $\mathrm{TiC}$. In the initial stage of ball milling, before $30 \mathrm{~h}$, under the impact of the grinding ball between the grinding pot, $\mathrm{W}$ powder was undergone severe plastic deformation due to the compression and shear of the grinding ball. The grain size became smaller, and the surface area increased. At this moment, toluene has not participated in the reaction but only acted as a process control agent to disperse the particles and reduce cold welding between powder particles. When the ball milling time was extended to $40 \mathrm{~h}$, under the catalytic action of the WC ball milling medium, toluene began to be catalytically decomposed, and the decomposed [C] began to react with nano-W powder to generate WC. And as the ball milling continued, the catalytic effect of the nano-WC generated during the ball milling would also accelerate the reaction. More $\mathrm{W}$ powder and $\mathrm{C}$ reacted to synthesize $\mathrm{WC}$ nanoparticles. In the process of alloying $\mathrm{W}$ and $\mathrm{C}$ to $\mathrm{WC}$, a very small part of $\mathrm{W}_{2} \mathrm{C}$ was produced. This phase was the intermediate 


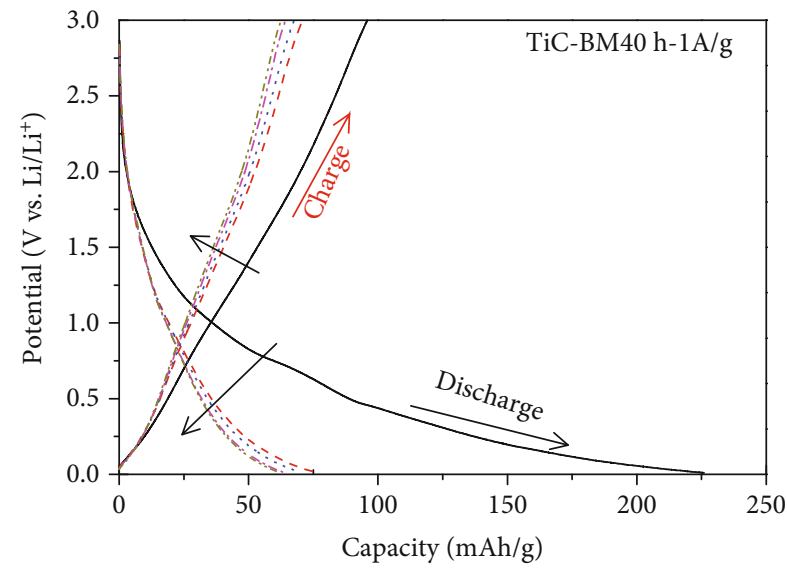

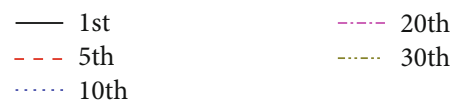

(a)

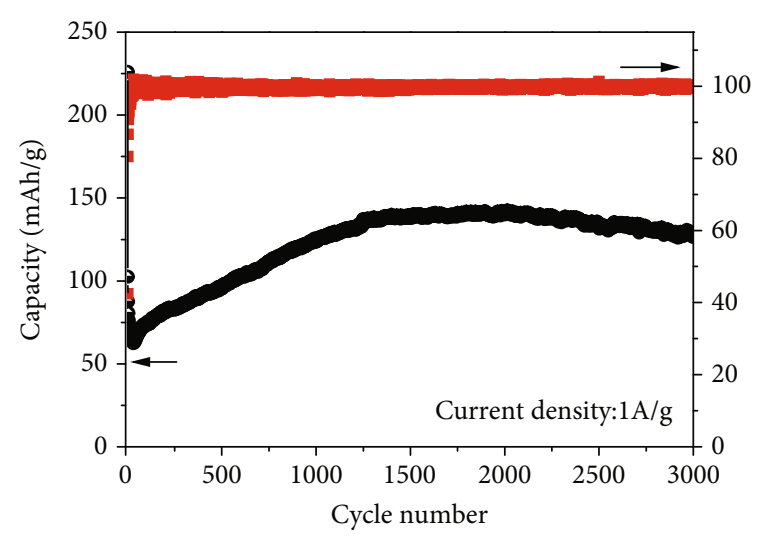

- TiC-BM40 h
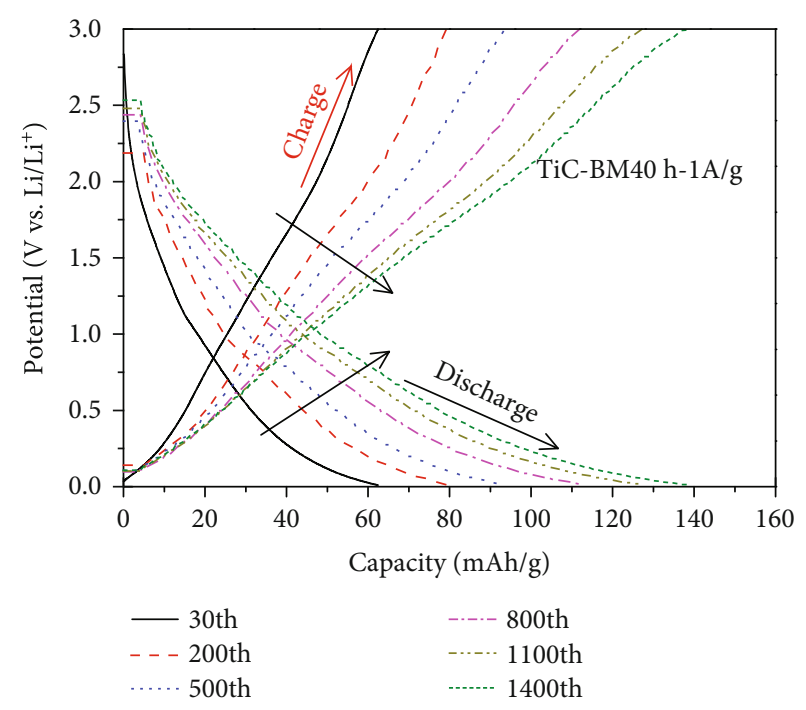

(b)

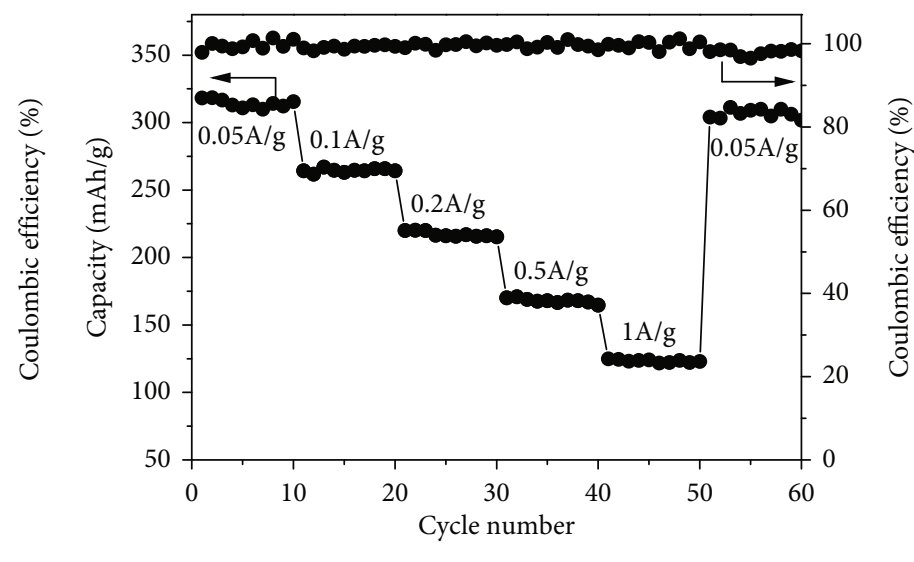

(d)

(c)

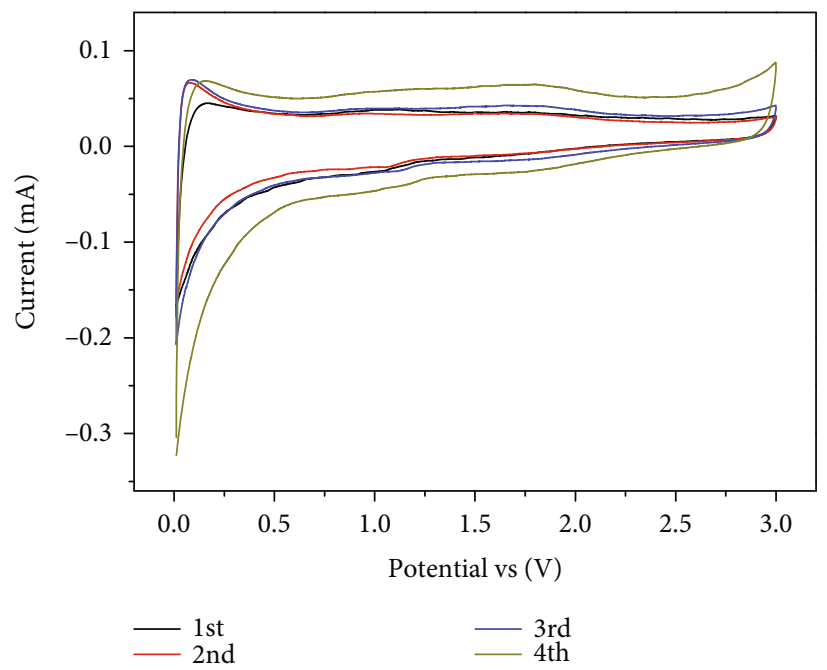

(e)

Figure 3: Charge and discharge performance of TiC-40: (a, b) galvanostatic charge and discharge curves for TiC, (c) cycling performance of TiC-40, (d) rate capability of TiC-40, and (e) CV curve of TiC-40. 
phase in the reaction. Due to the small content, it is not very obvious in the XRD spectrum. After $50 \mathrm{~h}$ of ball milling, $\mathrm{W}_{2} \mathrm{C}$ mesophase gradually changed to $\mathrm{WC}$ phase, so the powder after $50 \mathrm{~h}$ of ball milling was pure WC phase, the entire ball milling process was protected by Ar atmosphere, and there was no WHx and WOx phase after $50 \mathrm{~h}$ of ball milling. Compared with $\mathrm{TiC}$ ball milling powder, there were no other impurity components in WC after long-time alloying; therefore, no additional posttreatment process was needed.

The morphology evolution of WC particles after ball milling was similar to $\mathrm{TiC}$, as shown in Figure 5 . After $50 \mathrm{~h}$ of ball milling, the size of WC particles was small, about $10 \mathrm{~nm}$, and the shape was regularly spherical. After $60 \mathrm{~h}$ of ball milling, the size of WC particles increased slightly, with an average of $10 \mathrm{~nm}$, and the shape was basically spherical. After $70 \mathrm{~h}$ of ball milling, the size of WC particles became smaller to about $5 \mathrm{~nm}$. The reason for this change in the size of the ball mill particles was that during the ball milling process, the grain size of the powder was continuously reduced by the mechanical action of the ball milling process, and the particle size was also reduced simultaneously in the early stage of the ball mill, but since the ball milling time was longer, the surface energy of small particles was larger, and agglomeration will occur, which made the particle size gradually increased. As the ball milling process continued, the particle size change maintained a decreasing/increasing dynamic balance.

Compared with TiC ball milling, WC had higher ball milling efficiency. This may be due to the fact that WC had a slightly lower fracture toughness than TiC. During the ball milling process, the generated WC particles were easily hit repeatedly under the intense collision of the grinding balls for a long time. The impact of mill balls made the ball milling efficiency higher and the product particles finer. In addition, the dispersion of WC samples was better than that of TiC ball milling powder, but some amorphous inclusions similar to those of ball milling TiC nanoparticles can also be observed in the outer layer of the particles.

Although WC exists in the form of impurities in $\mathrm{TiC}$ ball milling, its content was not much. However, if the battery performance was poor, it will also have a greater impact on the overall $\mathrm{TiC}$ anode. Battery performance of the ballmilled WC nanopowder as the negative electrode was tested. The dispersion was uniform, and the phase was pure. It is similar to the WC impurities in the ball-milled $\mathrm{TiC}$ powder. A sample of ball milling for 60 hours was selected for testing. The charge and discharge performance of $\mathrm{WC}$ is shown in Figure 6.

The long-cycle charge and discharge result for $\mathrm{WC}$ is shown in Figure 6(a); the current density was $0.5 \mathrm{~A} / \mathrm{g}$. The trend of the discharge-specific capacity of ball-milled WC powder is similar to that of $\mathrm{TiC}$. The discharge capacity at the first cycle was $160.2 \mathrm{mAh} / \mathrm{g}$ and then quickly decreased to $62.3 \mathrm{mAh} / \mathrm{g}$. This large attenuation of discharge capacity was the irreversible lithium-ion insertion process of $\mathrm{WC}$ anode particles. As the charge-discharge cycle continued, the discharge capacity reached a minimum at 55 cycles, when the SEI layer tended to stabilize. After 55 cycles, the discharge capacity began to increase faster. At 900 times, it

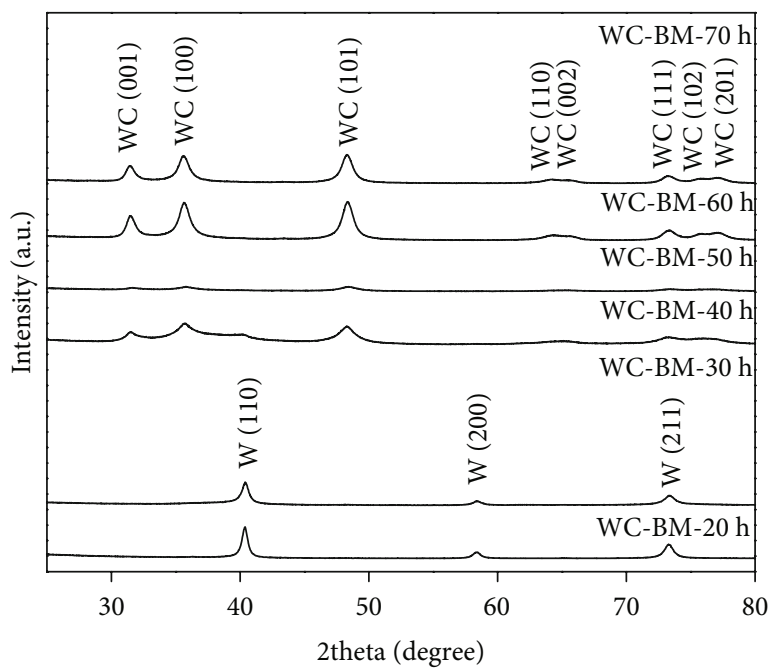

FIGURE 4: XRD patterns of WC powder ball milled for a series of time.

reached the extreme value of $142.5 \mathrm{mAh} / \mathrm{g}$ and then slowly decayed. The discharge capacity was kept at about $100 \mathrm{mAh} / \mathrm{g}$ when it is cycled to 2000 times. Compared with the ball-milled $\mathrm{TiC}$ anode, the initial discharge-specific capacity of the WC anode is lower, but the performance of the longer cycle was similar to TiC. The Coulombic efficiency is $46.8 \%$ at the first discharge and then gradually increased with the number of cycles to 55 times. To 2000 cycles, the Coulombic efficiency of the WC anode is maintained at about $98 \%$.

Figure 6(b) shows the cyclic voltammetry curve of the first 3 cycles ball milling WC; the scanning speed was $0.1 \mathrm{mV} / \mathrm{s}$. Similar to the cyclic voltammetric change of $\mathrm{TiC}$, as the number of cycles increased, the closed area in the curve of the ball-milled WC gradually decreased. In the first cycle, an insignificant reduction peak appeared at around $1.0 \mathrm{~V}$ in the curve. This reduction peak may represent toluene residues involved in lithium-ion deintercalation during ball milling. In the following cycle, this reduction peak disappeared, indicating that this is an irreversible reaction.

In order to study the rate performance of the ball-milled WC under variable current density conditions, the rate performance of the ball-milled WC was tested, as shown in Figure 6(c). It is similar to the system for testing the WC anode rate performance and $\mathrm{TiC}$. During the test, charge and discharge are 10 times at current densities of $0.05 \mathrm{~A} / \mathrm{g}$, $0.25 \mathrm{~A} / \mathrm{g}, 0.5 \mathrm{~A} / \mathrm{g}, 1 \mathrm{~A} / \mathrm{g}$, and $2 \mathrm{~A} / \mathrm{g}$, respectively, and finally return to $0.05 \mathrm{~A} / \mathrm{g}$ to test the rate performance. The initial discharge-specific capacity was $230 \mathrm{mAh} / \mathrm{g}$ and then gradually decayed to $90 \mathrm{mAh} / \mathrm{g}$ at $2 \mathrm{~A} / \mathrm{g}$. The current density returned to $0.05 \mathrm{~A} / \mathrm{g}$ and reached $230 \mathrm{mAh} / \mathrm{g}$ again. Compared with $\mathrm{TiC}$ anode, the cycle performance of ball-milled WC anode was not much reduced. Throughout the cycle, the Coulombic efficiency remained above $97 \%$.

It can be seen from the lithium battery test data that the ball-milled TiC and ball-milled WC have similar long cycle life. In terms of capacity performance, the reasons that affected the electrochemical performance of $\mathrm{TiC}$ anodes are 


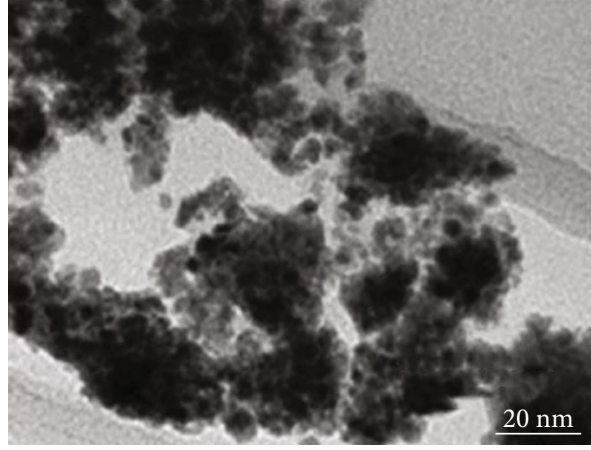

(a)

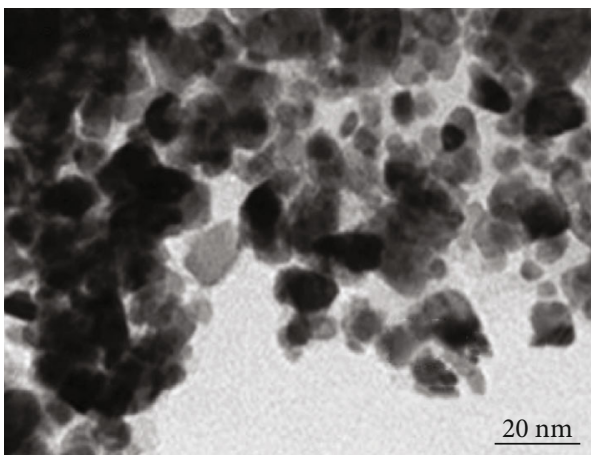

(c)

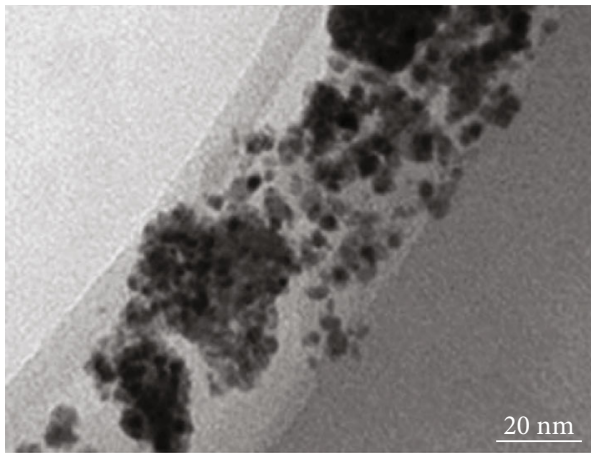

(e)

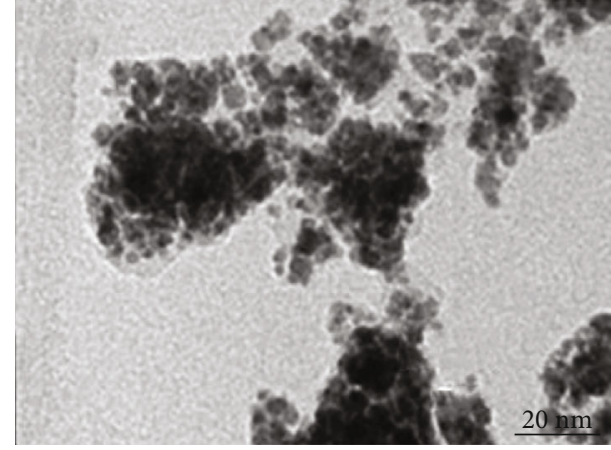

(b)

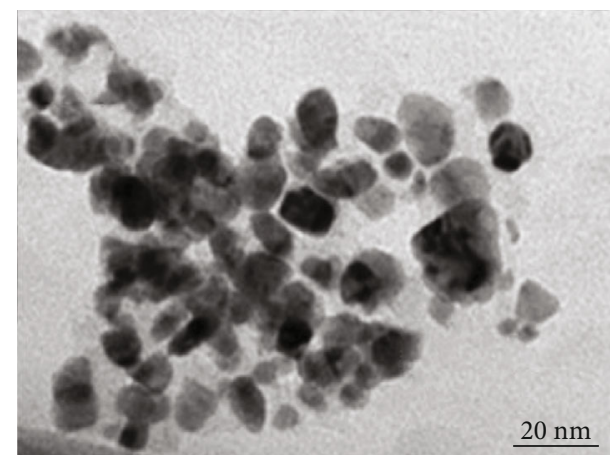

(d)

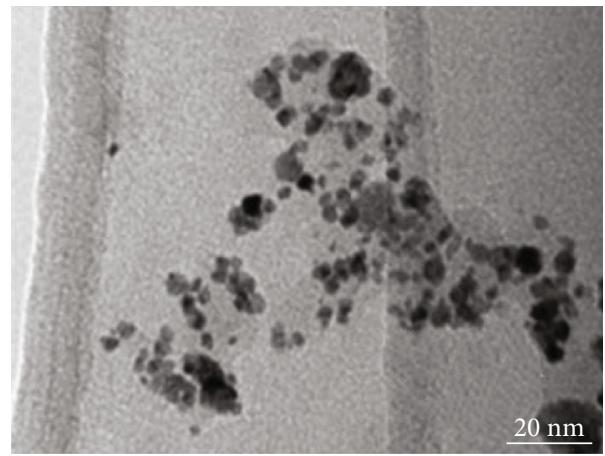

(f)

Figure 5

mainly the particle size and dispersion of nanoparticles and the content of WC impurities. To this end, the effect of different ball milling times was tested on the specific capacity of $\mathrm{TiC}$ discharge. Figure 7 shows the changes in the discharge capacity of $\mathrm{TiC}$ anodes with different initial ball milling times.

The WC impurities in the ball milling powder came from the collision and wear of the grinding ball during the ball milling process. The longer the milling time, the more WC impurities would be, and in the postprocessing process, WC would not decompose with the annealing treatment, and it had been kept in the ball mill powder until the end. In addition to the secondary ball milling after annealing, WC impurities were presented in all ball milling products to varying degrees. Therefore, the $\mathrm{TiC}$ samples after the initial ball milling were tested after $30 \mathrm{~h}, 40 \mathrm{~h}$, and $50 \mathrm{~h}$. As can be seen from the curve in Figure 7, the $40 \mathrm{~h}$ sample selected had a higher specific discharge capacity, followed by $30 \mathrm{~h}$.
The discharge capacity was $138.7 \mathrm{mAh} / \mathrm{g}$, the capacity of the $50 \mathrm{~h}$ sample is relatively low, and the specific discharge capacity for the first time is $107.7 \mathrm{mAh} / \mathrm{g}$. The reason may be in the two aspects of particle size and WC impurity content after ball milling. Compared with the $40 \mathrm{~h}$ sample, the $30 \mathrm{~h}$ sample has a shorter ball milling time and less WC impurity content, but the particle size of the powder was not fine enough. The corresponding smaller surface area restricted the diffusion of lithium ions, making its capacity curve rise slowly and the maximum value also lower, about $110 \mathrm{mAh} / \mathrm{g}$. There were more WC impurities stripped in $50 \mathrm{~h}$ sample, and the cycle life of WC was good, but the capacity is low, which could not only affect the cycle performance of the TiC anode but also reduce the overall discharge-specific capacity of the $\mathrm{TiC}$ anode. In addition, there were agglomeration phenomena in the ball-milled powder. The powder particles in the $50 \mathrm{~h}$ ball mill were finer. The larger surface area could produce relatively more 


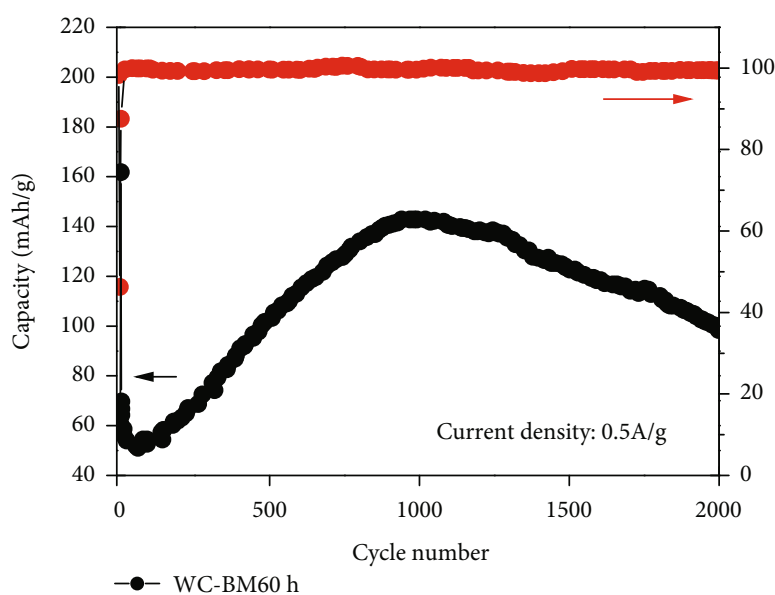

- WC-BM60h

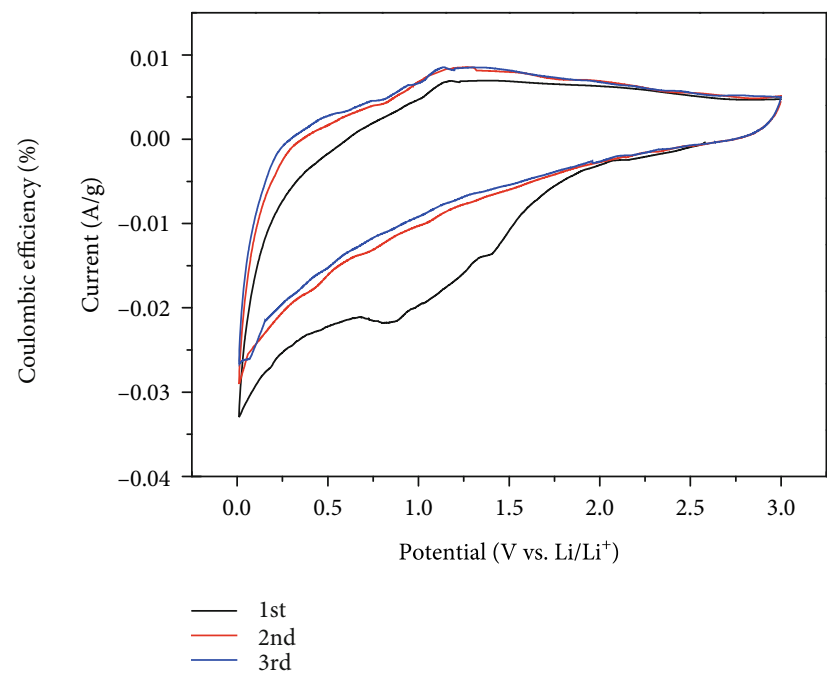

(b)

(a)

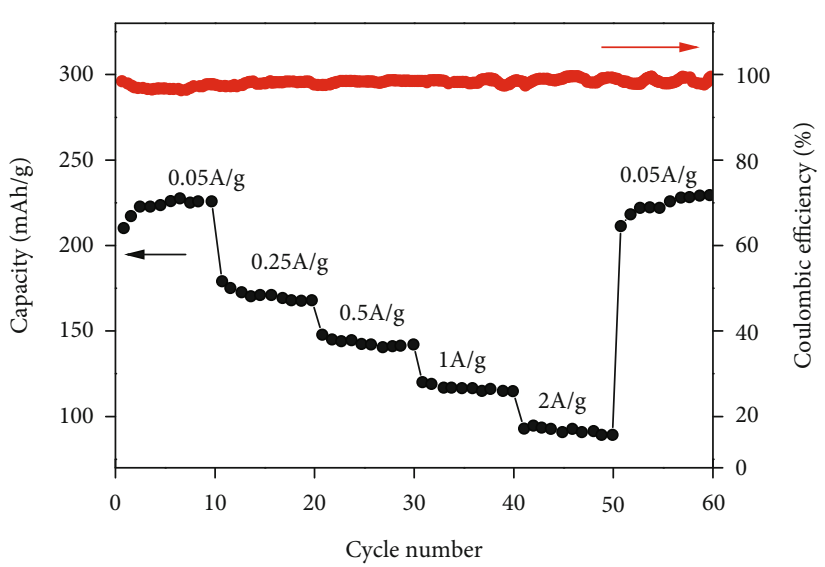

(c)

FIGURE 6: Charge and discharge performance of WC: (a) cycling performance of WC, (b) CV curve of WC, and (c) rate capability of WC.

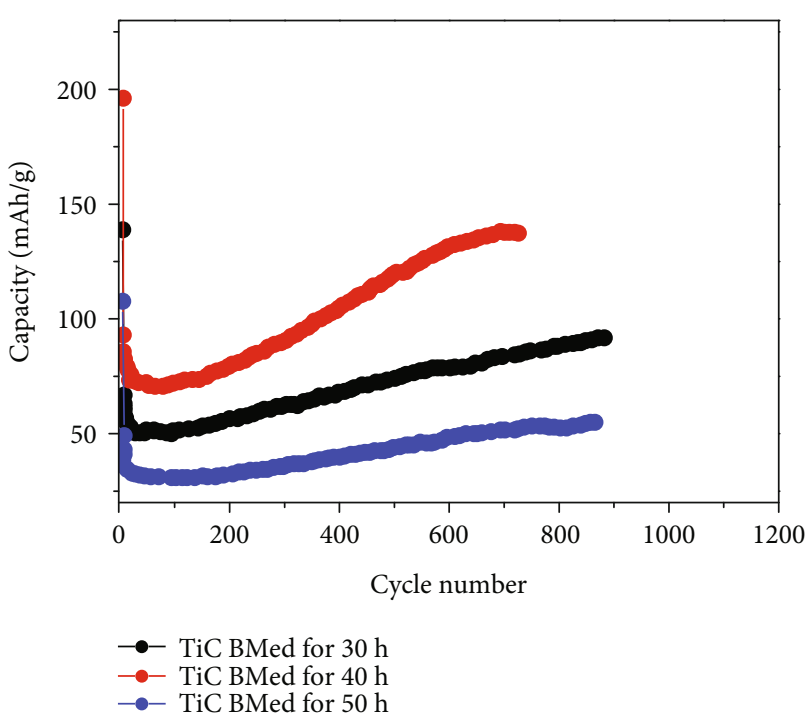

Figure 7: Cycling performance of TiC ball milled for $30 \mathrm{~h}, 40 \mathrm{~h}$, and $50 \mathrm{~h}$. agglomeration, and more WC impurities reduced its overall capacity. Therefore, the discharge capacity of TiC-50 anode was low.

\section{Conclusion}

In this paper, a high-energy ball milling method is used to prepare ultrafine $\mathrm{TiC}$ powder using toluene and pure titanium. After two-step ball milling, nanolevel TiC powder with higher purity is obtained, which is used as a negative electrode material to assemble a lithium-ion battery to test the lithium battery performance, the results show that the ballmilled TiC anode exhibits relatively excellent lithium battery performance, and its discharge capacity can still maintain $140 \mathrm{mAh} / \mathrm{g}$ after 3000 cycles. The small particle size and the toluene residue in the ball milling reaction affect the negative electrode particles during the charge and discharge process. Although the performance of $\mathrm{TiC}$ itself is not very good for lithium batteries, its long cycle performance is excellent, and the results show that it cannot only be used as a 
framework material for $\mathrm{Si}$ anode. TiC can also be used alone as a negative electrode material.

\section{Data Availability}

The raw/processed data required to reproduce these findings cannot be shared at this time as the data also forms part of an ongoing study. If you need to have access to some of the raw data, please contact the corresponding author.

\section{Additional Points}

Highlights. (1) Toluene is a carbon source to synthesize transition metal carbides. (2) Transition metal carbides are synthesized as anode materials for lithium batteries.

\section{Conflicts of Interest}

The authors declare that there is no conflict of interest regarding the publication of this paper.

\section{Acknowledgments}

We would like to thank the financial supports from the National Basic Research Program of China (973 Program) (Grant No. 2010CB731605 and No. 511CB808205), the National Science Fund for Distinguished Young Scholars (Grant No. 51025103), and the Natural Science Foundations of China (Grant No. 51272225, No. 51121061, and No. 51102206).

\section{References}

[1] S. Zhang, Q. Fan, Y. Liu et al., "Dehydration-triggered ionic channel engineering in potassium niobate for $\mathrm{Li} / \mathrm{K}$-ion storage," Advanced Materials, vol. 32, no. 22, article 2000380, 2020.

[2] Y. Zheng, T. Zhou, X. Zhao et al., "Atomic interface engineering and electric-field effect in ultrathin Bi2MoO6Nanosheets for superior lithium ion storage," Advanced Materials, vol. 29, no. 26, article 1700396, 2017.

[3] S. Zhang, Y. Zheng, X. Huang et al., "Structural engineering of hierarchical micro-nanostructured $\mathrm{Ge}-\mathrm{C}$ framework by controlling the nucleation for ultralong-life Li storage," Advanced Energy Materials, vol. 9, no. 19, article 1900081, 2019.

[4] G. Huang, S. Xu, Z. Xu, H. Sun, and L. Li, "Core-shell ellipsoidal $\mathrm{MnCo}_{2} \mathrm{O}_{4}$ anode with micro-/nano-structure and concentration gradient for lithium-ion batteries," ACS Applied Materials \& Interfaces, vol. 6, no. 23, pp. 21325-21334, 2014.

[5] Y. Hwa, W.-S. Kim, S.-H. Hong, and H. J. Sohn, "High capacity and rate capability of core-shell structured nano-Si/C anode for Li-ion batteries," Electrochimica Acta, vol. 71, pp. 201205, 2012.

[6] Y. Hwa, W.-S. Kim, B.-C. Yu, H. S. Kim, S. H. Hong, and H. J. Sohn, "Reversible storage of Li-ion in nano-Si/SnO $\mathrm{S}_{2}$ core-shell nanostructured electrode," Journal of Materials Chemistry A, vol. 1, no. 11, pp. 3733-3738, 2013.

[7] Q. Cen, Y. Jiang, R. Zhou, Y. Xu, and J. Wang, "Study on in situ synthesis of TiC particle reinforced iron matrix composite," Journal of Materials Engineering and Performance, vol. 20, no. 8, pp. 1447-1450, 2011.
[8] Z. Y. Zeng, J. P. Tu, Y. Z. Yang et al., "Nanostructured Si/TiC composite anode for Li-ion batteries," Electrochimica Acta, vol. 53, no. 6, pp. 2724-2728, 2008.

[9] J. Li, C. Yue, Y. Yu et al., "Si/Ge core-shell nanoarrays as the anode material for 3D lithium ion batteries," Journal of Materials Chemistry A, vol. 1, no. 45, pp. 14344-14349, 2013.

[10] C. Y. Tang, C. T. Wong, L. N. Zhang et al., "In situ formation of $\mathrm{Ti}$ alloy/TiC porous composites by rapid microwave sintering of Ti6Al4V/MWCNTs powder," Journal of Alloys and Compounds, vol. 557, pp. 67-72, 2013.

[11] F. Maglia, U. Anselmi-Tamburini, C. Deidda, F. Delogu, G. Cocco, and Z. A. Munir, "Role of mechanical activation in SHS synthesis of TiC," Journal of Materials Science, vol. 39, no. 16/17, pp. 5227-5230, 2004.

[12] J. Zhang, Q. Xue, and S. Li, "Microstructure and corrosion behavior of $\mathrm{TiC} / \mathrm{Ti}(\mathrm{CN}) / \mathrm{TiN}$ multilayer CVD coatings on high strength steels," Applied Surface Science, vol. 280, pp. 626-631, 2013.

[13] A. Jam, L. Nikzad, and M. Razavi, "TiC-based cermet prepared by high-energy ball-milling and reactive spark plasma sintering," Ceramics International, vol. 43, no. 2, pp. 2448-2455, 2017.

[14] N. Q. Wu, G.-X. Wang, J. M. Wu, Z. Z. Li, and M. Y. Yuan, "Investigation of $\mathrm{TiC}$ formation during ball-milling of elemental titanium and carbon," International Journal of Refractory Metals and Hard Materials, vol. 15, no. 5-6, pp. 289-293, 1997. 